\title{
ON A FIXED POINT THEOREM OF FISHER AND SESSA
}

\author{
GERALD JUNGCK \\ Department of Mathematics \\ Bradley University \\ Peoria, Illinois 61625, U.S.A. \\ (Received May 8, 1989)
}

ABSTRACT. A fixed point theorem of Fisher and Sessa is generalized by replacing the requirements of commutativity and nonexpansiveness by compatibility and continuity respectively.

KEY WORDS AND PHRASES. Common fixed points, commuting and compatible maps. 1980 AMS SUBJECT CLASSIFICATION CODE. 54H25.

\section{INTRODUCTION.}

In [1], Fisher and Sessa proved the following generalization of a theorem of Gregus [2].

THEOREM 1.1. ([1]). Let $T$ and $I$ be two weakly commuting mappings of a closed convex subset $C$ of a Banach Space $X$ into itself satisfying the inequality

$$
\|\mathrm{Tx}-\mathrm{Ty}\|<\mathrm{a}|| \mathrm{Ix}-\mathrm{Iy} \|+(1-a) \max \{\|\mathrm{Tx}-\mathrm{Ix}\|,\|\mathrm{Ty}-\mathrm{Iy}\|\} \quad \text { (1.1) }
$$

for all $x, y$ in $C$, where a $\varepsilon(0,1)$. If $I$ is linear and nonexpansive in $C$ and if $T(C) \subseteq I(C)$, then $T$ and $I$ have a unique common fixed point.

Sessa defined (see [1]) self maps $I$ and $T$ of a metric space ( $X, d)$ to be weakly commuting iff $d(I T x, T I x)<d(I x, T x)$ for $x \in X$. Subsequently, Jungck [3] defined two such self maps to be compatible iff whenever $\left\{x_{n}\right\}$ is a sequence in $x$ such that $T x_{n}, I x_{n}+t$ for some $t \varepsilon X$, then $d\left(I T x_{n}, T I x_{n}\right)+0$. Clearly, commuting maps are weakly commuting, and weakly commuting maps are compatible. [1] and [3] give examples which show that neither implication is reversible.

The purpose of this note is to show that Theorem 1.1 can be appreciably generalized by substituting compatibility for weak commutativity and continuity for the nonexpansive requirement. 


\section{RESULTS.}

The following fact from [3] will shorten the proof of our first result.

LEMMA 2.1 (Proposition 2.2, [3]). Let $f, g:(X, d) \rightarrow(X, d)$ be compatible.

1. If $f(t)=g(t)$, then $f g(t)=g f(t)$.

2. Suppose that $\lim _{n} f\left(x_{n}\right)=\lim _{n} g\left(x_{n}\right)=t$ for some $t$ in $x$.

(a) If $f$ is continuous at $t, 1 i m_{n} g f\left(x_{n}\right)=f(t)$.

(b) If $f$ and $g$ are continuous at $t$, then $f(t)=g(t)$ and $f g(t)=g f(t)$.

REMARK 2.1. We shall use $N$ to denote the set of positive integers and cl(S) to denote the closure of a set $S$.

PROPOSITION 2.1. Let $T$ and $I$ be compatible self maps of a metric space (X,d) with I continuous. Suppose there exist real numbers $r>0$ and a $\varepsilon(0,1)$ such that for all $x, y \in x$,

$$
d(T x, T y)<\operatorname{rd}(I x, I y)+a \max \{d(T x, I x), d(T y, I y)\} .
$$

Then $T w=I w$ for some $w \varepsilon X$ iff $A=n\left\{c l\left(T\left(K_{n}\right): n \varepsilon N\right\} \neq \emptyset\right.$, where $K_{n}=\{x \in X: d(T x, I x)<1 / n\}$.

PROOF. Suppose that Tw=Iw for some $w \varepsilon X$. Then $w \varepsilon K_{n}$ for all $n$ and thus $T w \varepsilon T\left(K_{n}\right) \subseteq c l\left(T_{n}\left(K_{n}\right)\right)$ for all $n ; i . e, T w \varepsilon A$.

Conversely, if $w \in A$, for each $n$ there exists $y_{n} \varepsilon T\left(K_{n}\right)$ such that $d\left(w, y_{n}\right)<1 / n$. Consequently, for each $n$ we can and do choose $x_{n} \varepsilon K_{n}$ such that $\mathrm{d}\left(w, T x_{n}\right)<1 / n$. So $T x_{n} \rightarrow w$. But since $x_{n} \varepsilon K_{n}, d\left(T x_{n}, I x_{n}\right)<1 / n$ and therefore $I x_{n}+w$. We have:

$$
\mathrm{Tx} \mathbf{n}_{\mathrm{n}}, \mathrm{Ix_{n }} \rightarrow \mathrm{w}
$$

Since I is continuous, (2.2) implies

$$
I T x_{n}, I^{2} x_{n} \rightarrow I w .
$$

Moreover, the compatibility of $I$ and $T$, and Lemma 2.1, and (2.2) yield

$$
d\left(T I x_{n}, I T x_{n}\right)+0 \text { and } T I x_{n}+I w \text {. }
$$

We now show that $T w=I w$. To this end observe that $d(T w, I w)<d\left(T w, T I x_{n}\right)$ $+d\left(T I x_{n} I w\right)$, and therefore, (2.4) implies

$$
d(T w, I w)<d\left(T w, T I x_{n}\right)+\varepsilon_{n} \text {, where } \varepsilon_{n}+0 \text { as } n+\infty \text {. }
$$

But with $x=w$ and $y=I x_{n},(2.1)$ says that

$$
d\left(T w, T I x_{n}\right)<r d\left(I w, I^{2} x_{n}\right)+a \max \left\{d(I w, T w), d\left(T I x_{n}, I^{2} x_{n}\right)\right\}
$$

for $n \in N$. So since $I^{2} x_{n}+I w$ by (2.3), we have

$$
d\left(T w, T I x_{n}\right)<a \max \left\{d(I w, T w), d\left(T I x_{n}, I^{2} x_{n}\right)\right\}+\delta_{n}
$$

for $n \varepsilon N$, where $\delta_{n}+0$. Consequently, in (2.5) we have

$$
d(T w, I w)^{n}<a \max \left\{d(I w, T w), d\left(T I x_{n}, I^{2} x_{n}\right)\right\}+\beta_{n},
$$

where $B_{n}=\varepsilon_{n}+\delta_{n}+0$ as $n \rightarrow \infty . \quad$ But $d\left(T I x_{n}, I^{2} x_{n}\right)+0$, by (2.3) and (2.4). Therefore, if $d(\mathrm{~T} w, \mathrm{I} w)>0,(2.6)$ implies that $d(\mathrm{~T} w, \mathrm{I} w)<a \mathrm{~d}(\mathrm{I} w, \mathrm{Tw})+\beta_{\mathrm{n}}$ for all sufficiently large $n$, and hence $d(T w, I w)<a d(I w, T w)\left(\beta_{n}+0\right)$. But then the assumption that $d(\mathrm{Tw}, \mathrm{Iw})>0$ demands that $a>1$, which contradicts the choice of a. 
We conclude that $d(T w, I w)=0$, and thus $I w=T w$.

THEOREM 2.1. Let $I$ and $T$ be compatible self maps of a closed convex subset $C$ of a Banach space $X$. Suppose that $I$ is continuous, linear, and that $T(C) \subseteq I(C)$. If there exists a $\varepsilon(0,1)$ such that for all $x, y \in C$

$$
\|\mathrm{Tx}-\mathrm{Ty}\|<\mathrm{a}\|\mathrm{Ix}-\mathrm{Iy}\|+(1-\mathrm{a}) \max \{|| \mathrm{Tx}-\mathrm{Ix}\|,\| \mathrm{Ty}-\mathrm{Iy} \|\},
$$

then $I$ and $T$ have a unique common fixed point in $C$.

PROOF. Let $K_{n}=\{x \in C:\|T x-I x\|<1 / n\}$ for $n \in N$. It is shown in [1], pages 24-26 - without appeal to the weak commutativity of $I$ and $T$ or the nonexpansiveness of $I$ - that by appeal to the convexity of $C$, the linearity of $I$, and property (2.1), we can infer that $A=n\left\{c 1\left(T\left(K_{n}\right)\right): n \varepsilon N\right\}$ is a $s i_{1}$, letton and therefore nonempty. Consequently, Proposition (2.1) then assures us that $I w=T w$ for some $w \in C$. We now show that $T w$ is a common fixed point of $I$ and $T$.

Since $I$ and $T$ are compatible and $I w=T w$, Lemma 2.1. implies that ITw=TIw. We then have $T^{2} w=T I w=I T w$, so that by $(2.1)$

$$
\begin{aligned}
\|\mathrm{TTw}-\mathrm{Tw}\| & <\mathrm{a}\|\mathrm{ITw}-\mathrm{Iw}\|+(1-\mathrm{a}) \max \{\|\mathrm{TTw}-\mathrm{ITw}\|,\|\mathrm{Tw}-\mathrm{Iw}\|\} \\
& <\mathrm{a}\|\mathrm{TTw}-\mathrm{Tw}\| ;
\end{aligned}
$$

thus, $T T w=T w$ since a $\varepsilon(0,1)$. By the above we then have $T w=T T w=I T w ; 1 . e ., T w 1 s$ a common fixed point of $T$ and $I$. That $T w$ is the only common fixed point of $I$ and $T$ follows immediately from (2.1).

In conclusion, we wish to present an example which shows that our Theorem 2.1 is indeed a generalization of Theorem 1.1. To effect this, the following result from [4] is helpful.

LEMMA 2.2. (Corollary 2.6, [4]). Suppose that $f$ and $g$ are continuous self maps of a metric space and that $f$ is proper. If $f x=g x$ implies $x=f x$, then $f$ and $g$ are compatible.

We remind the reader that a map $f: X+Y$ is proper iff $f^{-1}(C)$ is compact in $X$ when $C$ is compact in $Y$.

EXAMPLE 2.1. Let $X$ be the reals with the usual norm, $C=[0, \infty), I X=5 x / 2$ and $T x=$ $x+x\left(x^{2}+1\right)^{-1}$ for $x \in C$. Now $C$ is convex and closed, and $I, T: C \rightarrow C$ where $T(C)=$ $I(C)=C$ and $I$ is linear and continuous. Moreover, it is easy to see that the maximum value of $d T / d t$ on $c$ is 2,80 by the Mean Value Theorem we can say

$$
|T x-T y|<2|x-y|=\frac{4}{5}\left|\frac{5}{2} x-\frac{5}{2} y\right|=\frac{4}{5}|I x-I y|
$$

for all $x, y \in C$ so that condition (2.1) is satisfied. To see that $I$ and $T$ are compatible, note that both are continuous and that $I$ is clearly proper, so we can appeal to Lemma 2.2. Now $T x=I x$ implies that $x\left(3 x^{2}+1\right)=0$, so $x=0$. But $I(0)=0$ 
iff $x=0$; thus, $T$ and $I$ are Compatible by Lemma 2.2. Moreover, $|I x-I y|=\frac{5}{2}|x-y|$ and so $I$ is not nonexpansive. Finally, to see that $I$ and $T$ are not weakly commuting, observe that $\left|\mathrm{I}\left(\frac{1}{2}\right)-\mathrm{T}\left(\frac{1}{2}\right)\right|=7 / 20<21 / 41=\left|\operatorname{IT}\left(\frac{1}{2}\right)-\operatorname{TI}\left(\frac{1}{2}\right)\right|$.

\section{REFERENCES}

1. FISHER, B. and SESSA, S., On a Fixed Point Theorem of Gregus, Internat. J. Math. Math. Sci. 9 (1986), 23-28.

2. GREgUS, Jr., M., A Fixed Point Theorem in Banach Space, Boll. Un. Mat. Ital. 5 17-A (1980), 193-198.

3. JUNGCK, G., Compatible Mappings and Common Fixed Points, Internat. J. Math. Math. Sci. 9 (1986), 771-779.

4. JUNGCK, G., Common Fixed Points for Commuting and Compatible Maps on Compacta Proceedings of the American Mathematical Society 103 (1988), 977-983. 


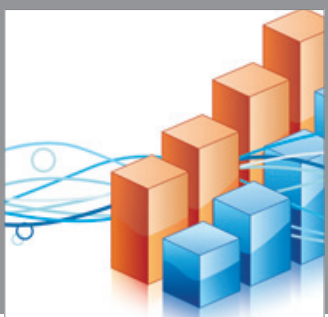

Advances in

Operations Research

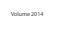

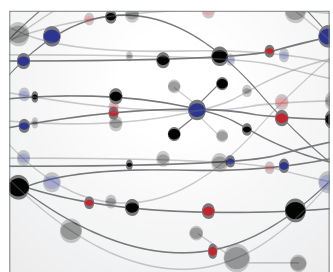

\section{The Scientific} World Journal
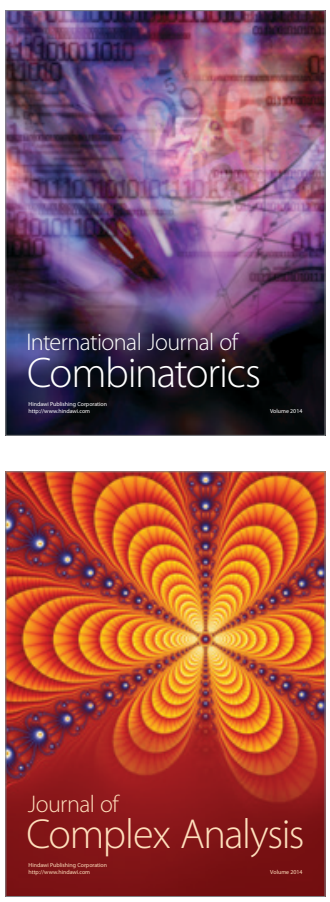

International Journal of

Mathematics and

Mathematical

Sciences
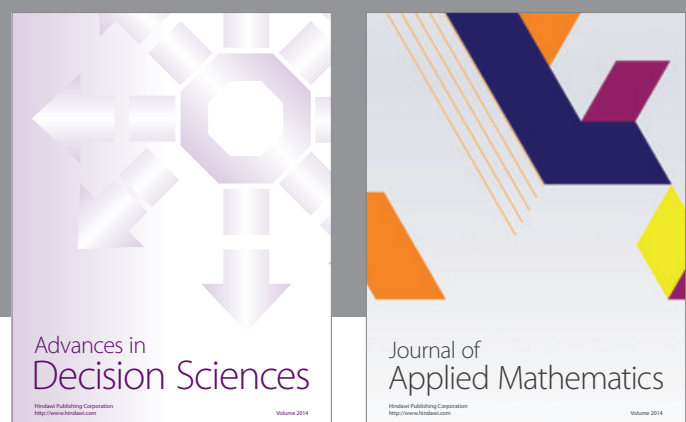

Journal of

Applied Mathematics
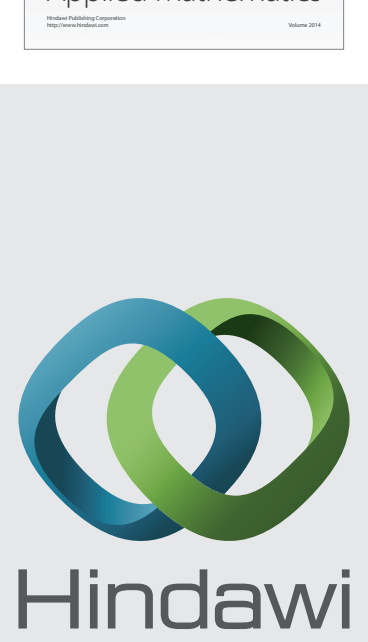

Submit your manuscripts at http://www.hindawi.com
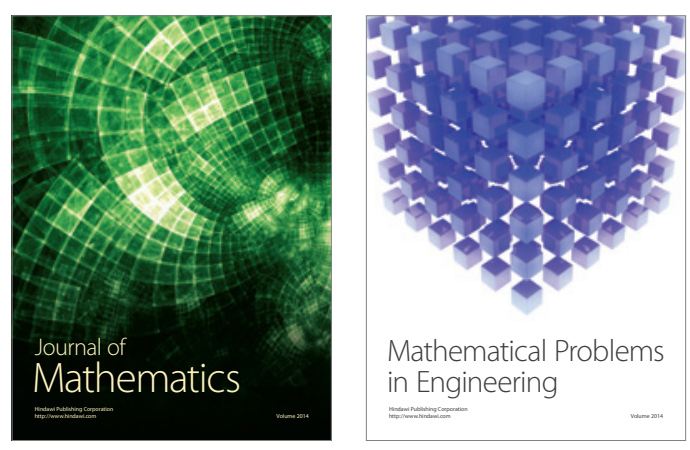

Mathematical Problems in Engineering
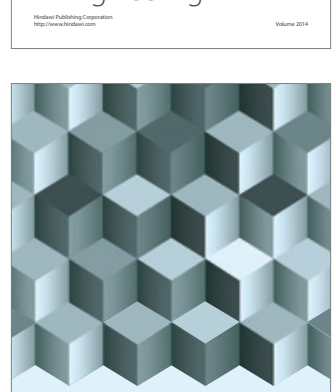

Journal of

Function Spaces
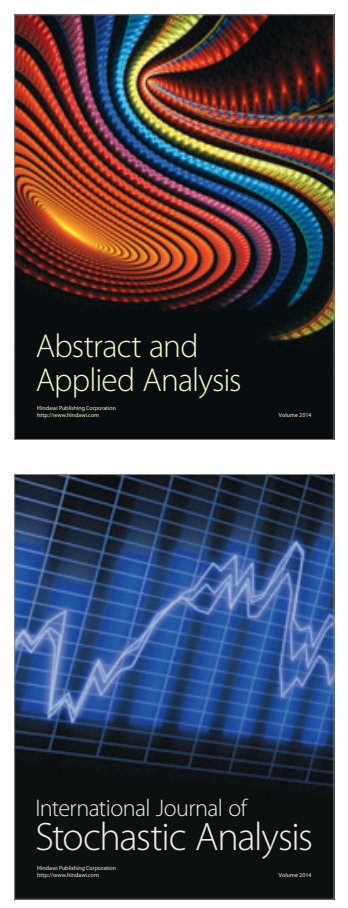

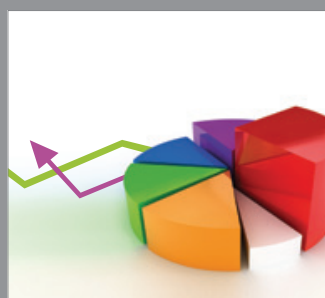

ournal of

Probability and Statistics

Promensencen
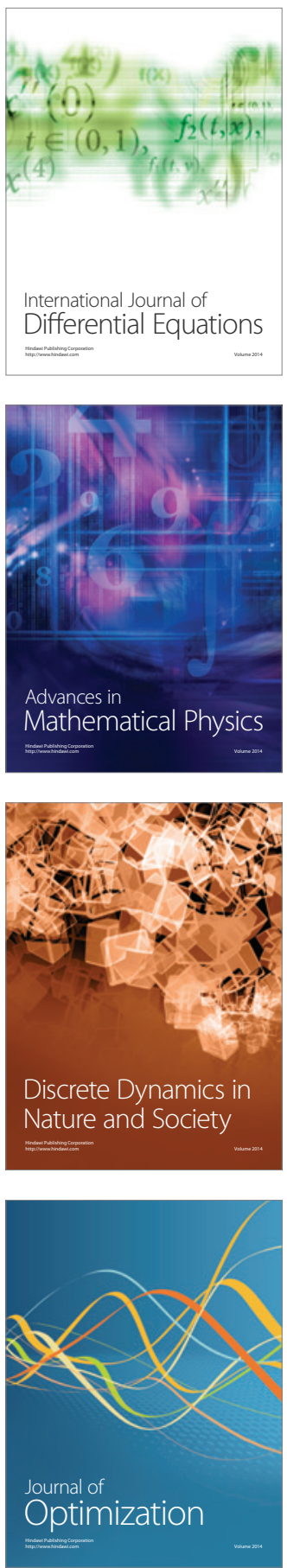